\title{
Diseño MECÁNICO DE PALAS PARA UN AEROGENERADOR DE 250W DE POTENCIA
}

\section{Mechanical design of blades for a wind turbines of $250 w$ power}

\author{
Joel la Paz Feliz ${ }^{a}$, Héctor Rodríguez Carr ${ }^{\mathrm{b}}$, Dinelson Rosa- \\ rio-Weeks ${ }^{c}$, Miguel Aybar-Mejía d ${ }^{\mathrm{d}}$, Deyslen Mariano-Hernández \\ y Alexeis Fernández Bonilla ${ }^{\mathrm{f}}$
}

Recibido: 8/6/21 - Aprobado: 13/6/21

Cómo citar: La Paz Feliz, J., Rodríguez Carr, H., Rosario-Weeks, D., Aybar Mejía, M., Mariano-Hernández, D., \& Fernández Bonilla, A. (2021). Diseño mecánico de palas para un aerogenerador de $250 \mathrm{w}$ de potencia. Ciencia, Ingenierías y Aplicaciones, 4(1), 25-55. DOI: https://doi.org/10.22206/cyap.2021.v4i1.pp25-55

\section{Resumen}

Las zonas rurales tienen una deficiencia de suministro eléctrico importante, ya que las comunidades están alejadas de las redes de transmisión eléctrica, por lo que el desarrollo se delimita apresuradamente en estos lugares. En zonas de este tipo, donde se dispone del recurso renovable del viento, la implantación de máquinas eólicas o aerogeneradores puede representar una solución viable e inmediata al problema energético de necesidades eléctricas básicas. Los aerogeneradores están planificados para áreas con fuertes corrientes de viento para generar la energía, y los mismos presentan de manera más eficiente una solución viable e inmediata, especialmente para el problema energético de las necesidades eléctricas básicas. El diseño experimental propuesto se basó en la literatura existen sobre el diseño de palas para generadores eólicos tomando en cuenta las condiciones locales de áreas rurales de la República Dominicana que tengan las condiciones de viento minima para que estas funcionen. Sin embargo, la indicación más significativa para seleccionar los materiales

\footnotetext{
a Área de Ingeniería. Instituto Tecnológico de Santo Domingo (INTEC), Santo Domingo, República Dominicana. Correo-e: 1072502@est.intec.edu.do

b Área de Ingeniería (INTEC). Correo-e: 1071540@est.intec.edu.do

c Área de Ingeniería (INTEC). Correo-e: dinelson.rosario@intec.edu.do

d Área de Ingeniería (INTEC). Correo-e: miguel.aybar@intec.edu.do

e Área de Ingeniería (INTEC).Correo-e: deyslen.mariano@intec.edu.do

f Área de Ingeniería (INTEC). Correo-e: alexeis.fernandez@intec.edu.do
} 
es la disponibilidad local. El diseño final presentado en las conclusiones es el resultado de una investigación teórica, una solución de ecuaciones matemáticas y un montaje experimental realizado en el Instituto Tecnológico de Santo Domingo, INTEC. Se espera que este trabajo sea un punto de partida para nuevas investigaciones relacionadas o mejoras al diseño actual en la República Dominicana.

Palabras clave: energía renovable; energía eólica; palas de aerogenerador; zonas rurales; aerogenerador; sostenibilidad; HAWT

\begin{abstract}
Rural areas have a significant electrical supply deficiency, as communities are far from electrical transmission grids, so development is hastily delimited in these locations. In areas of this type, where the renewable resource of the wind is available, the implementation of wind machines or wind turbines can represent a viable and immediate solution to the energy problem of basic electrical needs. Wind turbines are planned for areas with strong wind currents to generate ener$g y$, and they present a viable and immediate solution more efficiently, especially for the energy problem of basic electrical needs. The proposed experimental design was base on the existing literature on the design of blades for wind generators taking into account the local conditions of rural areas of the Dominican Republic that have the minimum wind conditions for them to work. However, the most significant indication for selecting materials is local availability. The final design presented in the conclusions results from a theoretical investigation, a solution of mathematical equations, and an experimental setup carried out at the Technological Institute of Santo Domingo, INTEC. This work is expected to be a starting point for new related research or improvements to the current design in the Dominican Republic.
\end{abstract}

Keywords: Renewable energy; wind energy; turbine blade; rural zone; wind turbine sustainability; HAWT 


\section{Introducción}

La energía eólica es una fuente de energía renovable que ha experimentado un enorme crecimiento en las últimas décadas (Cooperman, Eberle, \& Lantz, 2021). Los aerogeneradores de baja potencia trabajan bajo el principio de transformar la energía cinética de un recurso eólico en trabajo mecánico, que a su vez logra convertir esa energía mecánica en energía eléctrica a través de un generador (Junginger, Hittinger, Williams, \& Wiser, 2019). Debido a las condiciones climáticas que se presentan en la República Dominicana, la Comisión Nacional de Energía (CNE) establece que en el territorio nacional se tienen condiciones climáticas adecuadas para producir energía.

Las condiciones que presentan las áreas rurales son ideales para esta clase de proyectos, y en los estudios realizados en otras naciones se ve un impacto positivo dentro de este tipo de comunidades (Ngbara Touafio, Sanda, Malenguinza, M’Boliguipa, \& Mouangue, 2020).

Para una buena generación dentro de un área determinada se necesita una combinación de características de vientos que favorezcan la producción de energía (Saint-Drenan et al., 2020). Estos factores pueden ser desde la velocidad del viento promedio dentro de un determinado periodo de tiempo, los patrones de viento estacionales, entre otros (López-Villalobos, Rodríguez-Hernández, Martínez-Alvarado, \& Hernández-Yepes, 2021). En general, las ubicaciones con una velocidad media anual del viento de $7 \mathrm{~m} / \mathrm{s}$ o más son las más adecuadas para los sistemas de energía eólica conectados a la red de servicios públicos. Las aplicaciones de energía rural son usualmente viables a velocidades de viento más bajas ( 5 a $6 \mathrm{~m} / \mathrm{s})$, en algunos casos a velocidades de viento tan bajas como $4.5 \mathrm{~m} / \mathrm{s}$ (Elliott et al., 2001).

El diseño de aerogeneradores pequeños representa una solución efectiva y económica en las regiones del caribe (Costoya, de Castro, Santos, Sousa, \& Gómez-Gesteira, 2019). Es por lo que este documento se enfoca en el diseño mecánico de las palas aerogeneradores de eje horizontal (HAWT - Horizontal Axis Wind Turbine en inglés) con una capacidad de generación de $250 \mathrm{~W}$ y su impacto en el medio ambiente. 


\section{Metodología}

La estructuración de este artículo se basa en la presentación gradual de los conocimientos y deducciones basada en los datos arrojados mediante la investigación, empleando la metodología que se describe a continuación:

- Planteamiento para diseño y selección de los componentes mecánicos para palas mecánicas de aerogenerador de baja potencia de eje horizontal de $250 \mathrm{~W}$

- Búsqueda exhaustiva basada en palabras clave en distintas bases de datos como ScienceDirect e IEEE Xplore. La delimitación de palabras fue una combinación de las siguientes palabras: Energía eólica; Palas de aerogenerador; SolidWorks; Aerogenerador; HAWT.

- Filtrado de información: se realizó mediante una serie de pautas correspondientes a trabajos de investigación, trabajos de revisión, actas de congresos y libros de textos.

- Análisis y simulación de datos: se realizaron las simulaciones pertinentes, expuestas en el documento, utilizando softwares computacionales

\section{Dimensionamiento del aerogenerador}

\section{Descripción del sistema global}

La conformación de un aerogenerador está basada en la unión de una serie de elementos con la finalidad de poder convertir la energía mecánica en energía eléctrica aprovechando el viento, para luego poder alimentar las cargas (Xi et al., 2020).

Las principales partes de un aerogenerador incluyen los siguientes componentes:

- Rotor: pieza que gira de manera continua dentro de una máquina eléctrica, (generadores o motores eléctricos), encontrándose ubicada 
en su contraparte, el estator (Sugathapala, Boteju, Withanage, \& Wijewardane, 2020).

- El movimiento mecánico transferido por el viento es llevado hasta el generador gracias al eje de transmisión, que acopla la turbina al rotor del generador (Rauert, Herrmann, Dalhoff, \& Sander, 2016).

- Soporte o torre: estructura tubular que soportar la góndola y el rotor (Mühle, Adaramola, \& Sætran, 2017).

- La potencia eléctrica generada, es distribuida mediante un cableado y un circuito eléctrico que trasporta la energía desde su punto de generación hasta un banco de baterías, donde se almacena para su uso posterior (Rommel, Di Maio, \& Tinga, 2020).

- Las palas mecánicas o también conocidas como aspas de aerogeneradores es el componente encargado de recolectar la energía del viento (Denhof, Staar, Lütjen, \& Freitag, 2019). El diseño determina la cantidad de energía que se recolectará del viento (Moghadassian \& Sharma, 2020). Con el tiempo estas han ido mejorando la eficiencia, y la reducción de fricción con el eje (Micha, Mohan, \& Sivamani, 2017).

- En el mercado se encuentran una variedad de aerogeneradores de distintos tipos como los de eje vertical (Li, Maeda, Kamada, \& Mori, 2017), los de sistema de direccionamiento por veleta (Alsailani, Montazeri, \& Rezaeiha, 2021), con el empleo de distintos materiales dependiendo del área de utilización (Gugliani, Sarkar, Ley, \& Matsagar, 2021). Esta investigación se enfoca en la construcción de palas para aerogeneradores de eje horizontal con una potencia de $250 \mathrm{~W}$.

\section{Dimensionado del modelo propuesto}

El rotor del aerogenerador posee un diámetro de barrido de 1.30 $\mathrm{m}$, equivalente a $0.65 \mathrm{~m}(65 \mathrm{~mm})$ de longitud para cada una de las 3 palas. Se toma para condiciones estándares una eficiencia del generador 
eléctrico del $80 \%$ (Lara, Merino, Pavez, \& Tapia, 2011). La potencia ideal para la entrega es de $250 \mathrm{~W}$ por las condiciones de viento que se presenta en estas zonas (Garcia et al., 2019), esto es posible determinar gracias a una serie de modelados o sistemas de predicción de vientos dentro de un periodo de tiempo (Dong, Li, Gao, \& Zheng, 2020).

Para vientos de $10 \mathrm{~m} / \mathrm{s}$, con el tamaño de aerogenerador diseńado, solamente se puede extraer $491 \mathrm{~W}$ (tomando en cuenta el Límite de Betz), pero la energía total del viento en estas condiciones es de $823 \mathrm{~W}$, la cual no es completamente disponible. Las palas están disenadas con un TSR (Tip Speed Ratio) de 4, significando que la velocidad en la punta de las palas del rotor aumenta cuatro veces la velocidad del viento. A $10 \mathrm{~m} / \mathrm{s}$ el rotor gira a $588 \mathrm{RPM}$.

$\mathrm{Al}$ relacionar la potencia requerida $(313 \mathrm{~W})$ con la potencia disponible a $10 \mathrm{~m} / \mathrm{s}$ de viento $(491 \mathrm{~W})$, se determina un rendimiento de $63.63 \%$ y un factor de potencia del aerogenerador de $37.7 \%$. Mientras más alto este factor, mejor eficiencia posee el aerogenerador. Sin embargo, existe un punto máximo para este factor, el cual depende mayormente del diseño de las palas. Mediante los cálculos de referencia del libro Introduction to Wind Turbine Aerodynamics (Schaffarczyk, 2014), se procuró que este factor sea alcanzado alrededor de los $10 \mathrm{~m} / \mathrm{s}$. Los demás componentes mecánicos, como el eje de transmisión, carcasa, veleta y torre son diseñados con base en las referencias de varios autores de otros modelos similares de aerogeneradores HAWT de baja potencia. Para el diseño del eje, se utiliza los procedimientos del libro de Diseño en Ingeniería Mecánica de Shigley, (Budynas \& Nisbett, 2012). En la tabla 1 se presentan los requerimientos que se usaron para el diseño de las palas. 
Tabla 1. Requerimientos de diseño para el modelo de Aerogenerador propuesto

\begin{tabular}{ccc}
\hline Datos del aerogenerador a diseñar & & \\
\hline Diámetro de barrido & 1.30 & $\mathrm{~m}$ \\
\hline Longitud de palas & 0.65 & $\mathrm{~m}$ \\
\hline Área de barrido & 1.327 & \\
\hline Eficiencia del generador eléctrico & 0.80 & Adimensional \\
\hline Potencia de salida del diseño & 250 & $\mathrm{~W}$ \\
\hline Potencia de salida del rotor & 313 & $\mathrm{~W}$ \\
\hline Velocidad de viento nominal & 10 & $\mathrm{~m} / \mathrm{s}$ \\
\hline Rango de Viento & $2-12$ & $\mathrm{~m} / \mathrm{s}$ \\
\hline Condiciones ideales del aerogenerador a diseñar & & \\
\hline & 491 & $\mathrm{~W}$ \\
\hline Po & 823 & $\mathrm{~W}$ \\
\hline TSR & 0.6363 & Adimensional \\
\hline & 588 & $\mathrm{RPM}$ \\
\hline
\end{tabular}

Nota: las entradas son a $10 \mathrm{~m} / \mathrm{s}$ de viento. Datos asumidos por los autores 


\section{Diseño de partes mecánicas: rotor}

Es el componente que sostiene las palas y las conecta al eje principal de la máquina eólica. Los rotores vienen en muchas formas y configuraciones diferentes, principalmente dependiendo del tipo de generador utilizado y del diseño de las palas del rotor (Bernuzzi et al., 2021).

Las tres palas diseñadas utilizan un perfil aerodinámico NACA 4412 (Schmitz, 2020), con un TSR igual a 4, basado en los datos obtenidos de la herramienta Airfoil Tools. En cuanto el procedimiento aplicado se referencia en el libro de Introducción a la teoría de turbinas eólicas (Voneschen, 2009) conocido como "Método de las palas óptimamente diseñadas". El material es de madera Plywood debido a la disponibilidad local y su costo. El método para la fabricación de palas por costillas (Lima Menjívar, Orellana Ramos, \& Valencia Gómez, 2017), persigue el objetivo de mejorar la técnica de fabricación de la pala, por lo que se describe un procedimiento que facilite la aplicación de esta técnica de fabricación:

1. Usar una plantilla que contenga los perfiles de distintos tamaños y orientaciones correspondientes de la pala. En la plantilla cada perfil estará en un cuadro dentro de la plancha de madera, contendrá un agujero central por el que será atravesada una varilla de metal con extremos roscados para el apriete y lograr la estabilidad de la estructura.

2. Cortar en la plancha de madera las plantillas con una impresora láser u otra herramienta de corte. En el corte sale el perfil con su agujero.

3. Apilar en orden de tamaño (mayor a menor) los perfiles cortados. Se pegan con pegamento epóxido e irán tomando la orientación diseñada, y al mismo tiempo se crea un molde de madera con los cuadros cortados de donde sale el perfil, que sirve como molde para los perfiles de las otras aspas.

4. Introducir varilla de metal con puntas roscadas a través de los agujeros de los perfiles dentro del molde de madera. Enroscar en ambos extremos 
con tornillos para asegurar el apriete entre perfiles. Finalmente, se retira la pala desde la parte de abajo del molde de madera fabricado.

5. Realizar un recubrimiento de fibra de vidrio para añadir mayor resistencia a las palas, así como suavizar las superficies para mayor aerodinámica.

\section{Diseño de partes mecánicas: eje de transmisión}

El eje conecta el rotor con un acoplamiento al generador eléctrico. De esta manera se transmite el torque generado en el rotor hasta el generador eléctrico. El material es acero $1020 \mathrm{CD}$, para el diseño del eje de transmisión fueron utilizandos los libros de Diseño en Ingeniería Mecánica de Shigley (Budynas \& Nisbett, 2012) y Diseño de Maquinaria (Norton, 2009). Se hicieron los cálculos analíticos de cargas en el eje utilizando el software MD Solids.

\section{Datos de entrada:}

- Peso del Rotor (P1): $121 \mathrm{~N}$

○ Par de entrada = Par de salida: $5.00 \mathrm{Nm}$ (para un viento de $10 \mathrm{~m} / \mathrm{s}$ ).

- Factor de Diseño $\left(\mathrm{N}_{\mathrm{f}}\right): 1.5$

- Material: Acero $1020 \mathrm{CD}$

En la figura 2 se presenta los valores usados para hacer los análisis de fuerza cortante y momento flector del eje de transmisión. En la figura 3 se presenta las longitudes evaluadas para obtener las concentraciones de esfuerzo en el eje. Las condiciones de carga en el eje se tomarán como constantes, debido a las características del viento como:

- Los cambios de velocidad del viento ocurren de manera gradual y no repentinamente. Por lo que si la velocidad del viento disminuye la inercia del rotor mantiene la rotación de las palas a una velocidad que desacelera a ritmo constante. En caso contrario, si la velocidad del viento aumenta, se diseñó en la condición máxima de torque establecido $(10 \mathrm{~m} / \mathrm{s})$, por lo que cualquier otra velocidad no representará un peligro al eje diseñado. 
- Las palas del aerogenerador están diseñadas para rotar en un solo sentido, garantizándose por el mecanismo de orientación que alinea el aerogenerador en la dirección del viento.

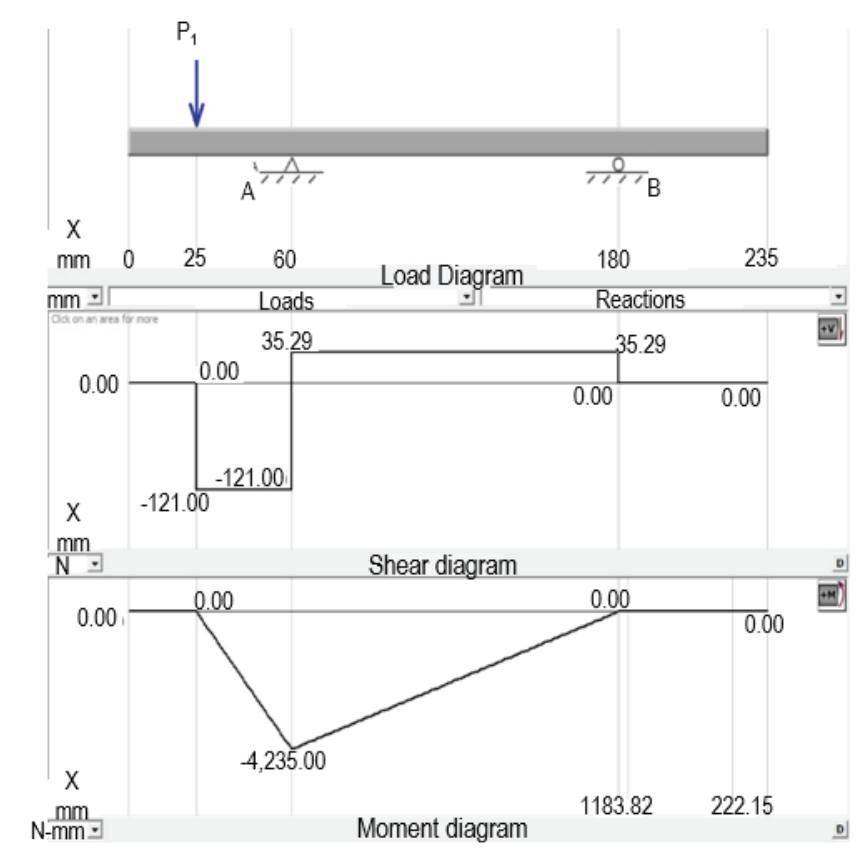

Figura 2. Diagramas de Fuerza Cortante y Momento Flector en el Eje de Transmisión utilizando el software MDSolids (Philpot, 2014)

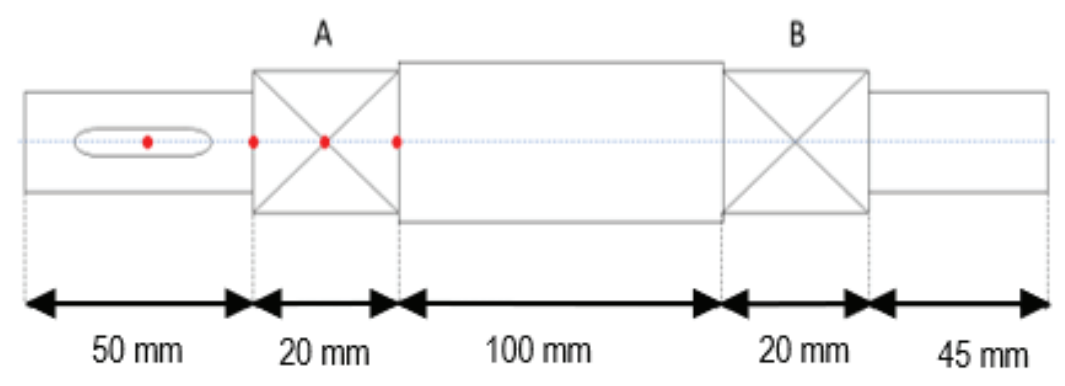

Figura 3. Diagrama representativo mostrando los puntos críticos del Eje de Transmisión para evaluar las concentraciones de esfuerzos y las dimensiones necesarias para evitar la falla del eje 
Se evalúa el Torque aplicado como un Torque Medio y el Momento Flector resultante como un Momento Alternante, donde el punto 2 es donde ocurre el Momento Flector Máximo en el eje. Usando la ecuación 6.8 del libro Diseño de Máquinas un enfoque integrado (Robert L Norton, 2011. pág. 422) se usó para determinar el diámetro requerido en el eje. Los escalones (cambios de sección), se tomaron con una relación típica de 1.20 , es decir, la relación entre el diámetro superior y el diámetro inferior en el eje.

La ecuación (1), se obtiene cuando en la ecuación 6.8 del libro Diseño de Máquinas un enfoque integrado (Robert L Norton, 2011. pág. 422) $\mathrm{Mm}=\mathrm{Ta}=0$, se reduce a lo siguiente:

$$
d=\left\{\frac{32 N_{f}}{\pi}\left[\frac{k_{f} M_{a}}{S_{e}}+\frac{\frac{\sqrt{3}}{2} k_{f s m} T_{m}}{S_{u t}}\right]\right\}^{\frac{1}{3}}
$$

\section{Análisis en el punto 2:}

- $\mathrm{Ma}=4235 \mathrm{~N}-\mathrm{mm}$

- $\mathrm{Tm}=5000 \mathrm{~N}-\mathrm{mm}$

$\circ \mathrm{N}_{\mathrm{f}}=1.5$

- Material: Acero $1020 \mathrm{CD}$

Los valores para $\mathrm{k}_{\mathrm{fs}}$ (flexión) $\mathrm{y}_{\mathrm{fsm}}$ (torsión), se asumen inicialmente de manera conservadora como 1.7 y 1.5 , respectivamente, para un filete de hombro bien redondeado $(\mathrm{r} / \mathrm{d}=0.1)$. El valor corregido de resistencia a la fatiga $S$, depende de diferentes constantes en base a las condiciones de trabajo

$$
S_{e}=C_{\text {carga }} C_{\text {tamaño }} C_{\text {sup }} C_{\text {temp }} C_{\text {conf }} S_{e^{\prime}}
$$

donde

$C_{\text {carga }}$ es el factor de carga, (Adimensional).

$C_{\text {tamaño }}$ es el factor de tamaño, (Adimensional).

$C_{\text {sup }}$ es el factor de superficie, (Adimensional).

$C_{\text {temp }}$ es el factor de temperatura, (Adimensional).

$C_{\text {conf }}$ es el factor de confiabilidad, (Adimensional).

$S_{e}$,es el Limite de resistencia a la fatiga, $\left(M P a \circ \mathrm{N} / \mathrm{mm}^{2}\right)$. 
Para los aceros se cumple que, si la resistencia última del material $\mathrm{S}_{\mathrm{ut}}$ es menor a $1400 \mathrm{MPa}$, entonces el Límite de resistencia a la fatiga es igual a $0.5 \mathrm{~S}_{\mathrm{ut}}$ (Ecuación 4.5a, Norton, 2009, p. 260). Entonces:

$$
S_{e^{\prime}}=0.5 \mathrm{~S}_{\mathrm{ut}}=0.5(460)=230 \mathrm{MPa}
$$

Los factores de corrección se determinaron de la siguiente manera:

- Factor de Carga

Para casos de torsión pura o flexión, $C_{\text {carga }}=1.000$

\section{- Factor de Tamaño}

El diámetro del eje a diseñar debe estar entre 2.79 y $51 \mathrm{~mm}$ (Ecuación 6.20, Budynas \& Nisbett, 2012, p. 275). Tomando una estimación inicial del diámetro como $10 \mathrm{~mm}$, el factor de tamaño es:

$$
\mathrm{C}_{\text {tamaño }}=1.24 \mathrm{~d}^{-0.107}=1.24(10)^{-0.107}=0.9692
$$

- Factor de Superficie

Para el acabo superficial de Maquinado o Laminado en frío.

$$
\begin{aligned}
\mathrm{a} & =4.51, \mathrm{~b}=-0.265 \\
\mathrm{C}_{\text {sup }}=\mathrm{aS}_{\text {ut }}^{\mathrm{b}} & =(4.51)(460)^{-0.265}=0.8883
\end{aligned}
$$

- Factor de Temperatura

La temperatura de trabajo puede asumirse como $50^{\circ}$, tomando en cuenta el generador eléctrico. (Con la tabla 6-4, Budynas \& Nisbett, 2012, p. 278).

$$
\mathrm{C}_{\text {temp }}=1.008
$$

\section{- Factor de Confiabilidad}

Con una confiablidad del $95 \%$, se utilizó la Tabla 6-5 (Budynas \& Nisbett, 2012, p. 279). 


$$
\mathrm{C}_{\text {conf }}=0.868
$$

Por tanto, el valor corregido de esfuerzo a la fatiga es tomado como:

$$
\begin{gathered}
\mathrm{S}_{\mathrm{e}}=\mathrm{C}_{\text {carga* }} \mathrm{C}_{\text {tamańo* }} \mathrm{C}_{\text {sup }} \mathrm{C}_{\text {temp }}{ }^{*} \mathrm{C}_{\text {conf }} \mathrm{S}_{\mathrm{e}^{\prime}} \\
\mathrm{S}_{\mathrm{e}}=(1.000)(0.9692)(0.8883)(1.008)(0.868)(230)=173 \mathrm{MPa}
\end{gathered}
$$

Introduciendo los valores en la ecuación (1):

$$
\mathrm{d}=\left\{\frac{32(1.5)}{\pi}\left[\frac{(1.7)(4235)}{173}+\frac{\frac{\sqrt{3}}{2}(1.7)(5000)}{460}\right]\right\}^{1 / 3}=\mathbf{9 . 6 0} \mathbf{~ m m}
$$

En la tabla A-17 p. 999 del libro de Budynas y Nisbett (2012) para las dimensiones estándar, el diámetro comercial del eje en su punto crítico es de $10 \mathrm{~mm}$. Con una relación de escalones típico de 1.20, el diámetro siguiente es de $12.00 \mathrm{~mm}$, y el anterior es de $9 \mathrm{~mm}$. Las dimensiones en el otro extremo del eje serán las mismas determinadas para el punto crítico.

Después de confirmadas las dimensiones críticas, se seleccionó las dimensiones como $20 \mathrm{~mm}, 25 \mathrm{~mm}$ y $30 \mathrm{~mm}$ para los diámetros en los puntos 1, 3, 4, por razones de conveniencia para los cojinetes que se seleccionan con base en la Carga Nominal de Catálogo $\mathrm{C}_{10 .}$, usando ecuación 11.3, de Budynas y Nisbett (2012, p. 551), para una confiabilidad del $90 \%$.

$$
C_{10}=F_{D}\left(\frac{L_{D}}{L_{10}}\right)^{1 / a}
$$

donde:

$C_{10}$ es la Carga Nominal de Catálogo, (Adimensional).

$F_{D}$ es la Carga Real del cojinete, (Adimensional). 
$L_{D}$ es la vida útil de diseño, (Adimensional).

$L_{10}$ es la vida útil nominal, (Adimensional).

$a$ es el parámetro del tipo de cojinete, $\left(\mathrm{MPa} o \mathrm{~N} / \mathrm{mm}^{2}\right)$.

Del software MDSolids, se obtuvieron las cargas reales en los cojinetes como las reacciones en los apoyos A y B, y sus magnitudes son $156 \mathrm{~N}$ y $35 \mathrm{~N}$, respectivamente, seleccionando un solo tipo de cojinete con base en el apoyo A. Determinando la vida útil por

$$
L_{D}=60 n L_{D}
$$

Donde:

$n$ es la velocidad nominal del cojinete, (rev/min o RPM).

$L_{D}$ es la vida útil de diseño en el tiempo, (horas).

Los libros establecen una vida útil en diez millones de revoluciones $\left(10^{6}\right)$. El parámetro para el tipo de cojinete es:

$\circ \quad \mathrm{a}=3$, para cojinete de bolas.

○ $a=10 / 3$, para cojinete de rodillo o cónico.

Utilizando la tabla 11.4 (Budynas \& Nisbett, 2012, p. 559), se toma en cuenta las horas de operación efectiva (a máxima capacidad) como 8 horas. Para máquinas para servicio de 8 h, establecemos una vida útil en tiempo de 10000 horas. La velocidad nominal de los cojinetes es de 600 RPM. Introduciendo en la ecuación (c).

$$
C_{10}=(156)\left(\frac{(600)(10000)(60)}{10^{6}}\right)^{1 / 3}=912 \mathrm{~N}
$$




\section{Comentarios}

Los cojinetes de referencia, para un diámetro de $25 \mathrm{~mm}$, tienen cargas nominales del orden de los $\mathrm{kN}$, por lo que las necesidades de diseño están cubiertas en esta parte. Adicionalmente, los cojinetes poseen retenedores de aluminio para mantener la fijación del eje a pesar de las vibraciones y movimientos erráticos.

\section{Diseño de partes mecánicas: otros componentes}

\section{CARCASA}

Es el componente que protege los elementos, con suficiente espacio como para añadir elementos adicionales de control, posee dos partes, las cuales están unidas por dos bisagras y una cerradura de pestillos de palanca que evita que se abra durante operación.

\section{VELETA}

Es el dispositivo de orientación situado en la prolongación del eje del rotor. Están constituidas por una superficie plana metálica o de madera, sobre la que el viento ejerce una presión en el momento en que no están orientadas paralelamente en la dirección de este, provocando un par de giro que orienta la máquina (Gato, Maduro, Carrelhas, Henriques \& Ferreira, 2021).

Se necesitan dos soportes: 1) El que une la carcasa con el tubo de dirección, 2) El que une el tubo de dirección con el soporte de veleta. Ambos implicarían el uso de maquinado y soldadura. La plancha de madera puede extraerse de las mismas que se usen para las palas.

\section{TORRE}

Consta de una base en forma de trípode atornilladas al suelo. Estas patas son soldadas a un tubo de acero, hueco, en el que se inserta otro tubo, igualmente hueco, dando la altura completa para la máquina eólica. En el extremo superior, se conecta un soporte giratorio (con cojinete), el cual permite la sujeción de la carcasa a la torre, así como la rotación de este. 


\section{Resultado de las simulaciones}

\section{ROTOR}

\section{- Cálculo de potencia y velocidad de giro}

Con los parámetros especificados del aerogenerador, aplicamos las fórmulas para la potencia de salida y velocidad de giro en el rotor (Voneschen, 1989; 2009).

$$
P_{w}=c_{p} P_{w, \max }
$$

donde

$P_{w}$ es la potencia entregada por el rotor, (W).

$C_{p}$ es el factor de potencia del rotor, (Adimensional).

$P_{\text {u,max }}^{p}$ es la potencia teórica máxima posible entregable por el rotor, (W).

$$
P_{w, \max }=0.37 F v^{3}
$$

Donde:

F es el área de barrido por las palas, $\left(\mathrm{m}^{2}\right)$

$\mathrm{v}$ es la velocidad del viento que atraviesan las palas, $(\mathrm{m} / \mathrm{s})$

$$
n=(60 \vee \lambda) /(\pi D)
$$

Donde:

$\lambda$ es el Tip Speed Ratio (TSR), (Adimensional)

$\mathrm{D}$ es el diámetro de barrido de las palas, (m)

$n$ el número de revoluciones por minuto a las que gira el rotor, (RPM)

En la tabla 2 se presenta los valores de velocidad del viento correlacionado con la velocidad angular del eje del rotor en revoluciones por minuto $(\mathrm{RPM})$ y la potencia aproximada de salida de este para esas condiciones. 
Tabla 2. Comparación de velocidad de viento y RPM generados en el rotor del aerogenerador

\begin{tabular}{|c|c|c|}
\hline Velocidad de Viento $(\mathrm{m} / \mathrm{s})$ & RPM del Rotor & Potencia de salida (W) \\
\hline 0.00 & 0 & 0.0 \\
\hline 0.50 & 29 & 0.0 \\
\hline 1.00 & 59 & 0.2 \\
\hline 1.50 & 88 & 0.8 \\
\hline 2.00 & 118 & 1.9 \\
\hline 2.50 & 147 & 3.8 \\
\hline 3.00 & 176 & 6.5 \\
\hline 3.50 & 206 & 10.3 \\
\hline 4.00 & 235 & 15.4 \\
\hline 4.50 & 264 & 21.9 \\
\hline 5.00 & 294 & 30.1 \\
\hline 5.50 & 323 & 40.0 \\
\hline 6.00 & 353 & 51.9 \\
\hline 6.50 & 382 & 66.0 \\
\hline 7.00 & 411 & 82.5 \\
\hline 7.50 & 441 & 101.4 \\
\hline 8.00 & 470 & 123.1 \\
\hline 8.50 & 500 & 147.6 \\
\hline 9.00 & 529 & 175.3 \\
\hline 9.50 & 558 & 206.1 \\
\hline 10.00 & 588 & 240.4 \\
\hline 10.50 & 617 & 278.3 \\
\hline 11.00 & 646 & 320.0 \\
\hline 11.50 & 676 & 365.6 \\
\hline 12.00 & 705 & 415.4 \\
\hline 12.50 & 735 & 469.5 \\
\hline 13.00 & 764 & 528.2 \\
\hline 13.50 & 793 & 591.5 \\
\hline 14.00 & 823 & 659.7 \\
\hline 15.00 & 881 & 811.4 \\
\hline
\end{tabular}

Nota: se asume un factor de potencia del rotor de $37.7 \%\left(C_{p}=0.377\right)$. El TSR del diseño es de 4. 


\section{Cálculos de esfuerzo de flexión y visualización de resistencia al viento de las palas}

Se utilizaron las herramientas de Solidworks Flow Simulation para observar el comportamiento del viento en un rango de 5 a $12 \mathrm{~m} / \mathrm{s}$ cuando atraviesa una pala. De esta simulación, se importaron los datos de los esfuerzos generados sobre la superficie de la pala y se determinó la deflexión máxima resultante en Solidworks Stress Analysis, cono se muestra en la tabla 3.

Tabla 3. Resultados simulaciones de viento y esfuerzo

\begin{tabular}{ccc}
\hline \multicolumn{3}{c}{ Simulación: Túnel de viento de las palas } \\
\hline $\begin{array}{c}\text { Velocidad del viento } \mathrm{m} / \mathrm{s} \\
(\mathrm{km} / \mathrm{s})\end{array}$ & $\begin{array}{c}\text { Presión relativa } \\
\text { máxima }(\mathrm{Pa})\end{array}$ & Deflexión máxima $(\mathrm{mm})$ \\
\hline $5.00(18.0)$ & 37.80 & 0.0288 \\
\hline $6.00(21.6)$ & 54.60 & 0.0403 \\
\hline $6.00(25.2)$ & 73.90 & 0.0541 \\
\hline $6.00(28.8)$ & 97.60 & 0.0697 \\
\hline $6.00(32.4)$ & 123.80 & 0.0878 \\
\hline $6.00(36.0)$ & 150.30 & 0.1080 \\
\hline $6.00(39.6)$ & 183.30 & 0.1300 \\
\hline $6.00(43.2)$ & 217.70 & 0.1550 \\
\hline
\end{tabular}

\section{Comentarios}

- La deflexión de las palas es mínima por la acción del viento. Esto es debido al material seleccionado de madera Plywood, el cual es bastante rígido y resistente.

\section{- Curva característica de las palas en función del TSR}

Con el documento sobre Introducción a la teoría de las turbinas eólicas (Voneschen, 2009), es posible realizar los cálculos aproximados para determinar el factor de potencia de la pala dependiendo del TSR (figura 7). Los datos de los coeficientes de arrastre y sustentación para distintos ángulos de ataque del perfil NACA 4412 fueron extraídos de 
la página web de AirfoilTools (AirfoilTools, 2020). De acuerdo con la teoría de turbinas eólicas, el punto de la pala donde se estima el factor de potencia de la pala completa es en el $72 \%$ de su longitud total, partiendo desde el centro del rotor (Voneschen, 2009). En la figura 5 se presenta la relación de velocidad de la punta (TSR) frente al coeficiente de potencia (CP) y tabla 4 se presenta los valores simulados para los coeficientes de las palas propuestas.

\section{Curva Característica de Palas}

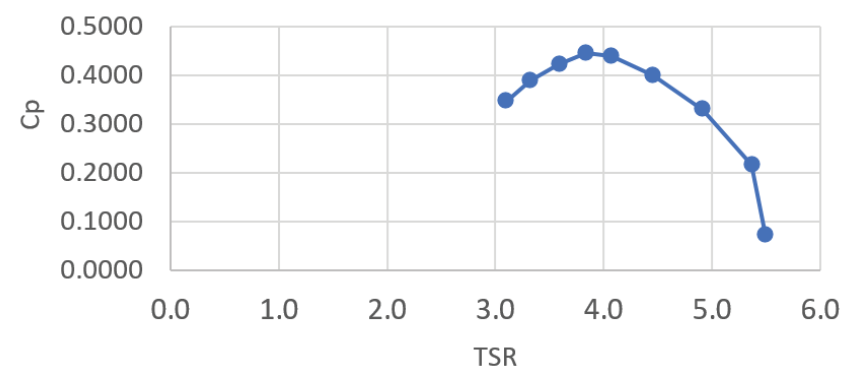

Figura 5. Curva característica de palas del modelo propuesto

En la tabla 4 se puede observar que para los ángulos se obtienen un coeficiente de potencia mayor como se muestra a continuación:

Tabla 4. Resultados de curva característica de palas del modelo propuesto

\begin{tabular}{ccccc}
\hline $\mathrm{r} / \mathrm{R}=0.72$ & $\mathrm{r}=0.42 \mathrm{~m}$ & $\delta=5.8^{\circ}$ & $\mathrm{t}=0.0720 \mathrm{~m}$ & $\mathrm{z}=3$ \\
\hline$\alpha$ & $\mathrm{Ca}$ & $\mathrm{Cw}$ & $\mathrm{TSR}$ & $\mathrm{Cp}$ \\
\hline $0^{\circ}$ & 0.4938 & 0.01005 & 5.5 & 0.0707 \\
\hline $2^{\circ}$ & 0.7038 & 0.01097 & 5.4 & 0.2173 \\
\hline $4^{\circ}$ & 0.9127 & 0.01256 & 4.9 & 0.3299 \\
\hline $6^{\circ}$ & 1.1122 & 0.01444 & 4.5 & 0.4002 \\
\hline $8^{\circ}$ & $\mathbf{1 . 2 7 9 9}$ & $\mathbf{0 . 0 1 6 7}$ & $\mathbf{4 . 1}$ & $\mathbf{0 . 4 3 9 2}$ \\
\hline $10^{\circ}$ & $\mathbf{1 . 3 2 7 5}$ & $\mathbf{0 . 0 2 5 5 5}$ & 3.8 & $\mathbf{0 . 4 4 5 7}$ \\
\hline $12^{\circ}$ & 1.3641 & 0.03917 & 3.6 & 0.4224 \\
\hline $14^{\circ}$ & 1.4180 & 0.05407 & 3.3 & 0.3897 \\
\hline $16^{\circ}$ & 1.4362 & 0.07501 & 3.1 & 0.3459 \\
\hline
\end{tabular}




\section{TORRE}

Se simuló la respuesta de la torre del aerogenerador propuesto aplicando una carga de $900 \mathrm{~N}$, sobre la columna principal, el cual representa el peso total del sistema a sostener (Véase la figura 6).

(a)
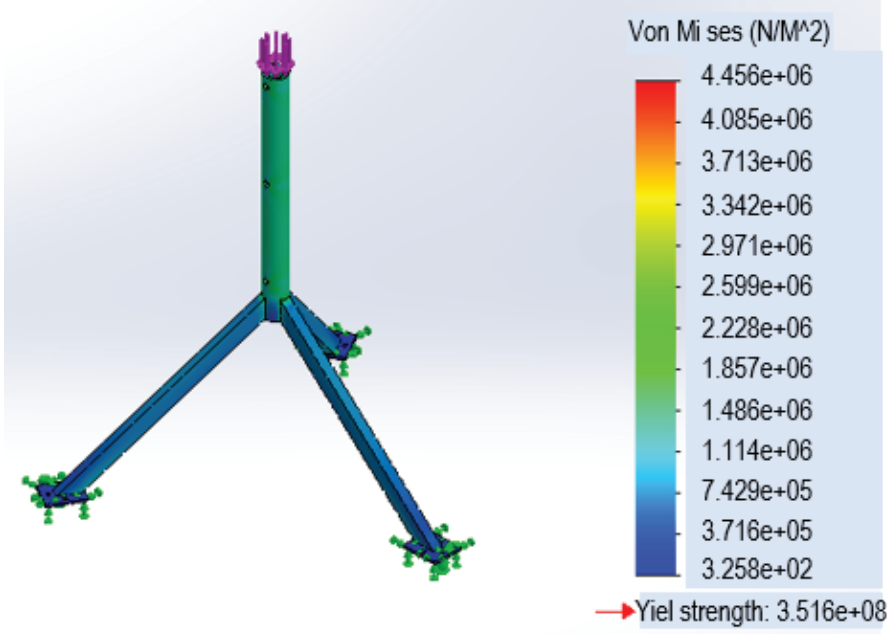

(b)

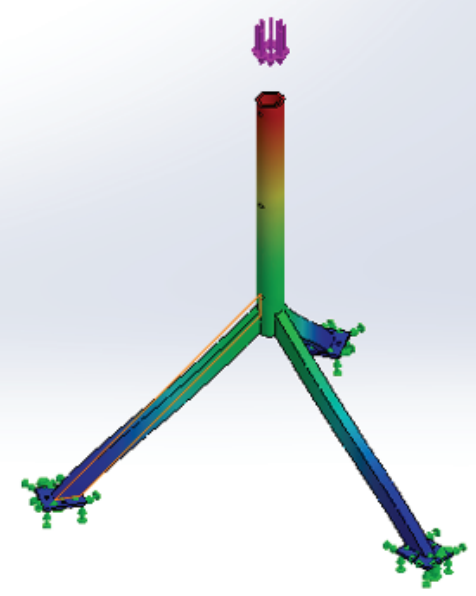

URE (mm)

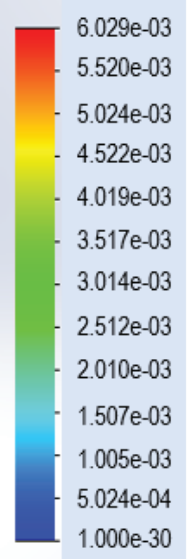

Figura 6. Simulación de cargas y resultados de esfuerzo (a) y compresión (b) para la base de trípode del aerogenerador propuesto. Simulación realizada por los autores en el software SolidWorks 


\section{Simulación de rotación de las palas del rotor a $600 \mathrm{RPM}$}

En el programa de simulación Flow Simulation no es posible determinar la velocidad de giro que produce una corriente de viento sobre las palas del aerogenerador. Sin embargo, puede hacerse el proceso inverso, es decir, girar las palas del aerogenerador para conocer el flujo de viento que produce. Es por esto que en el experimento a la inversa para comprobar que si las palas giran a 588 RPM, entonces el viento generado es de $10 \mathrm{~m} / \mathrm{s}$ y es posible producir $250 \mathrm{~W}$. Exitosamente, se cumplieron los resultados esperados. En la figura 6 se presenta el modelo propuesto de las palas y en figura 7 y figura 8 los resultados simulados de las palas en el software SolidWorks.
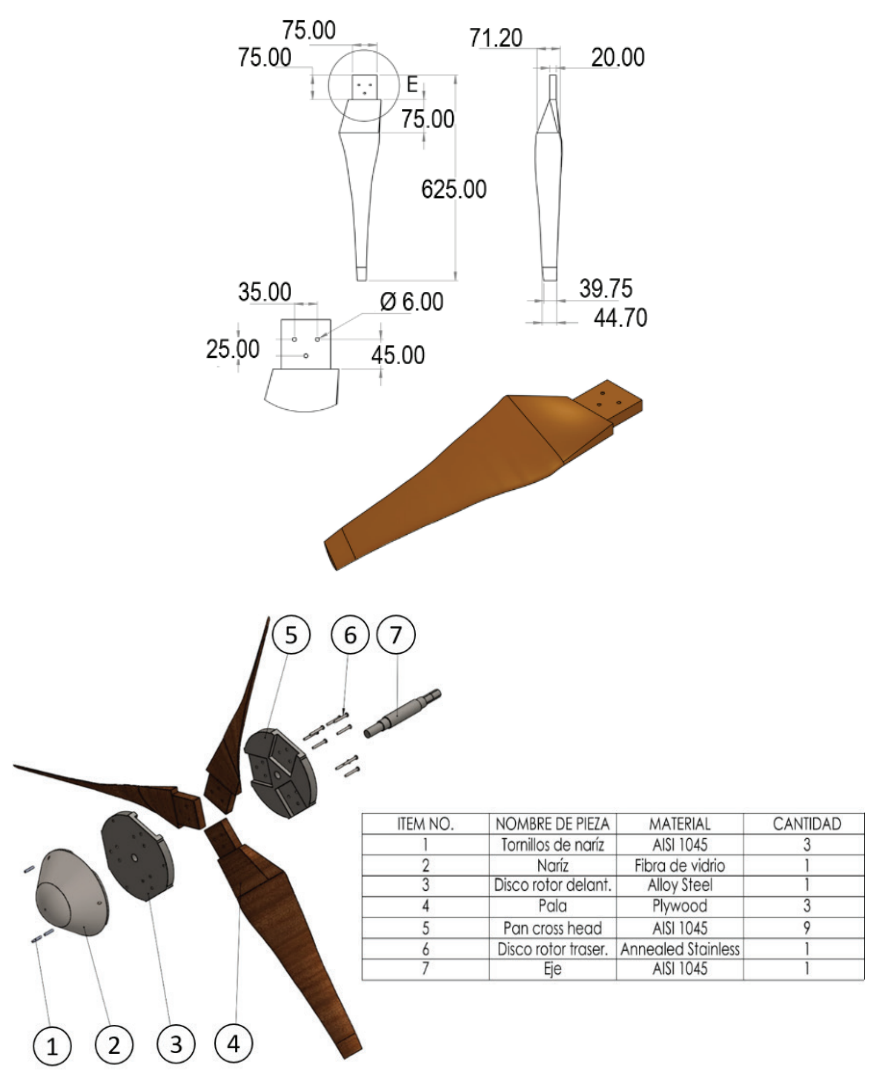

Figura 6. Esquemáticos de las palas diseñadas. Presentado las dimensiones de las palas en milímetros y como se acoplan al rotor 

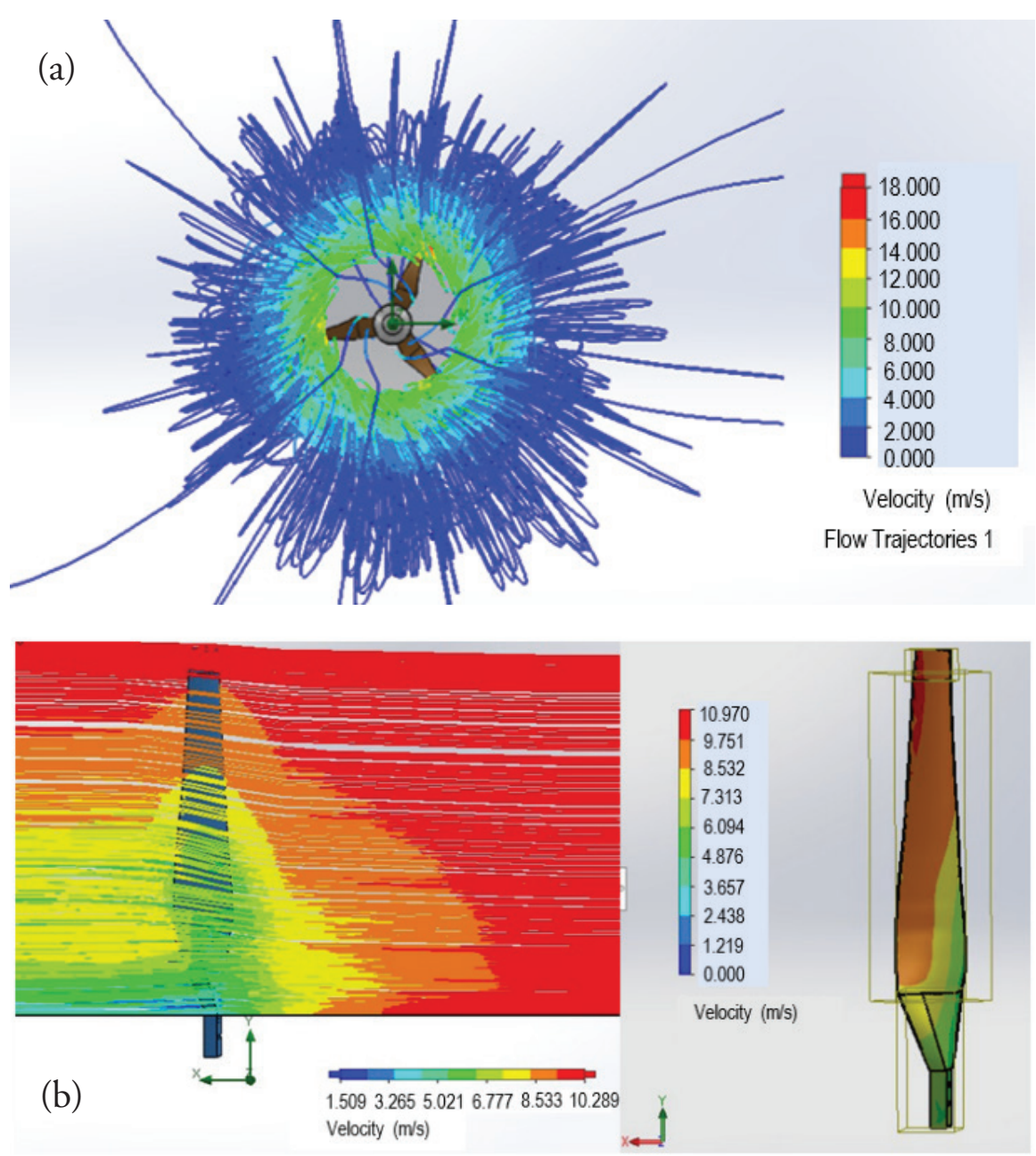

Figura 7. (a) Resultados de simulación de rotación de las Palas a 600 RPM y (b) resultados de simulación de pala sometida a un túnel de viento de $10 \mathrm{~m} / \mathrm{s}$ 


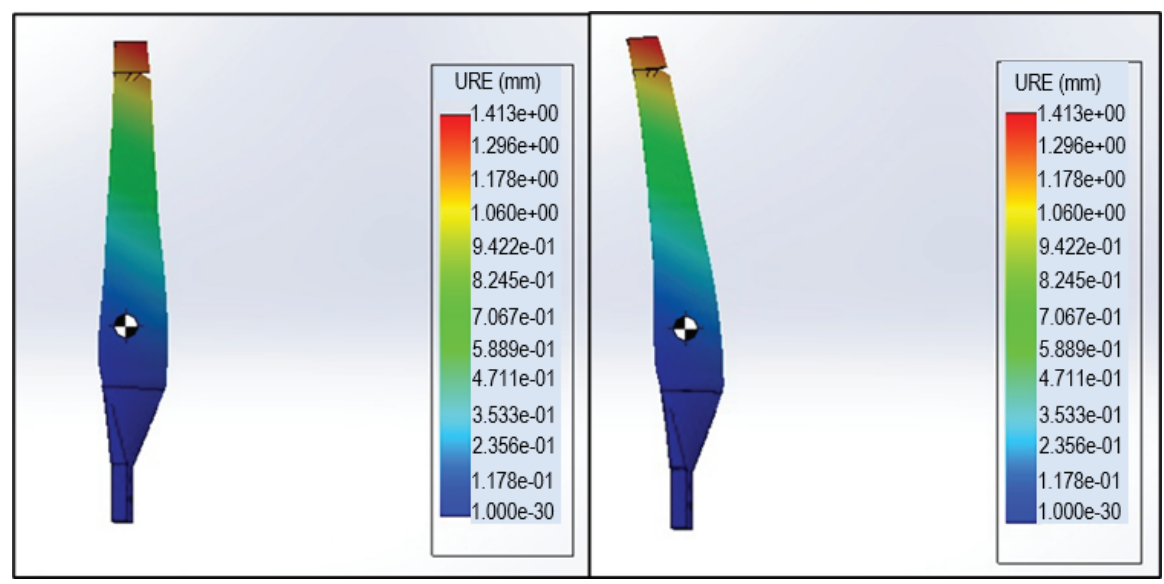

Figura 8. Resultados de simulación de esfuerzos en una pala para una velocidad de viento de $10 \mathrm{~m} / \mathrm{s}$

\section{Análisis económico}

Los costos de este proyecto se estimaron con base en los precios ofertados en ferreterías y talleres industriales locales. Cabe destacar que estos precios no son definitivos, varían según la inflación monetaria, por lo que al momento de construir este proyecto es recomendable revisar esta tabla de precios como referencia, se sugiere mantener los gastos al mínimo.

Se debe resaltar que este prototipo está conceptualizado con la finalidad de que pudiera ser elaborado por cualquier persona que desee implementar estas técnicas para construir sus propias palas mecánicas.

En el mercado moderno se pueden encontrar distintos tipos de modelos de aerogenerador de baja potencia de 400W, $1000 \mathrm{~W}$ a un costo menor que el propuesto, estos oscilan entre 200 y 300 dólares. Sin embargo, la diferencia fundamental es la durabilidad y resiliencia de las partes presentadas en este diseño, con una vida útil de 20 años (Herrmann, Rauert, Dalhoff, \& Sander, 2016) en las condiciones de trabajo con alta exposición solar, humedad, alta velocidad de los vientos y lluvias. Es por esto que se recomienda como una opción tanto para la República Dominicana como en toda la región del Caribe que presente 
las mismas condiciones. Como agregado se pueden aumentar el tamaño de las palas, para obtener mayor capacidad de producción de energía.

A continuación, presentaremos los precios analizando los materiales necesarios para su construcción como se expone en la tabla 5.

Tabla 5. Presupuesto estimado de materiales y servicios para el proyecto en desarrollo

\begin{tabular}{|c|c|c|}
\hline Material o servicio & $\begin{array}{l}\text { Precio estimado para } \\
\text { consumidor (RD\$) }\end{array}$ & $\begin{array}{l}\text { Precio estimado para } \\
\text { consumidor (USD) }\end{array}$ \\
\hline $\begin{array}{l}\text { Plancha de madera Plywood } \\
\text { 9mm (3/8) Okume }\end{array}$ & 1000 & 17.14 \\
\hline Eje de acero & $600-900$ & $10.28-15.42$ \\
\hline $\begin{array}{l}\text { Tubo redondo de acero } \\
\text { (Torre) }\end{array}$ & 600 & 10.28 \\
\hline Plancha de acero & $14000-16000$ & $240-274$ \\
\hline $\begin{array}{l}\text { Tubo rectangular hueco de } \\
\text { acero }\end{array}$ & 700 & 12.00 \\
\hline $\begin{array}{c}\text { Cojinetes radiales x } 2 \\
\text { unidades }\end{array}$ & $300 \times 2$ & $5.14 \times 2$ \\
\hline Tornillería & $6-100 \times$ cada pieza & $0.10-1.71 \times$ cada pieza \\
\hline Varilla roscada x 1 unidad & 80 & 1.37 \\
\hline $\begin{array}{c}\text { Pegamento epóxido x } 1 \\
\text { unidad. }\end{array}$ & 900 & 15.42 \\
\hline $\begin{array}{c}\text { Paquete de fibra de vidrio de } \\
1 / 2 " \text { × } 2 \text { unidades }\end{array}$ & 140 & 2.40 \\
\hline $\begin{array}{l}\text { Servicio de maquinado } \\
\text { CNC }\end{array}$ & $\begin{array}{c}\text { Depende de la pieza } \\
500-5000\end{array}$ & $\begin{array}{c}\text { Depends on the piece } 8.57- \\
85.69\end{array}$ \\
\hline Servicio de impresora 3D & $\begin{array}{l}\text { Depende de las piezas. } \\
\text { Costo por kg 18-30 USD }\end{array}$ & $\begin{array}{c}\text { It depends on the parts. Cost } \\
\text { per kg 18-30 USD }\end{array}$ \\
\hline Servio de cortado láser & 1000 & 17.14 \\
\hline $\begin{array}{l}\text { Servicio de recubrimiento de } \\
\text { fibra de vidrio }\end{array}$ & 500 & 8.57 \\
\hline Precio Total Estimado & $30,000 \mathrm{RD} \$$ & 520 USD \\
\hline $\begin{array}{c}\text { Nota: tasa del dólar fue } \\
\text { tomada como } 58.35 \mathrm{RD} \$ \\
\text { (julio 2020) }\end{array}$ & & \\
\hline
\end{tabular}




\section{Impacto medioambiental}

La madera Plywood, aunque es elaborada en un proceso de compactación caliente no queda exenta de sufrir efectos de descomposición cuando se encuentra a la intemperie, por esta razón, para esta aplicación se utilizó un recubrimiento de fibra de vidrio para brindar una protección total de la madera por medio del encapsulamiento de las palas en este tipo de resina.

La fibra de vidrio convencional sin recubrimientos especiales tiene un tiempo de descomposición aproximado de 50 años bajo condiciones de cuidados especiales (Rahimizadeh, Kalman, Fayazbakhsh, \& Lessard, 2019), cuando es expuesto a los rayos ultravioleta este tiempo de vida decrece aproximadamente a 4 años. La solución para el alargamiento de la vida útil de la resina es un aditamento de protección llamado Gelcoat, el cual es un material parecido a la resina, pero con bases de poliéster insaturado y resinas epóxicas (Rafiee et al., 2019). El Gelcoat ofrece durabilidad y resistencia al estrés mecánico brindándole un tiempo de vida aproximado de 15 ańos expuesto a la intemperie (Pomázi \& Toldy, 2021). Al culminar el tiempo de vida estimado de dichos aerogeneradores se han estipulado varios proyectos encaminado a la reutilización de estos materiales, volviendo todavía más amigables al planeta estas maquinarias (Joustra, Flipsen, $\&$ Balkenende, 2021). En la tabla 6 se presenta el plan de mantenimiento básico del aerogenerador.

Tabla 6. Plan de mantenimiento de aerogenerador HAWT 250W diseñado

\begin{tabular}{ll}
\hline Actividad & Periodicidad \\
\hline Inspección y limpieza general & Anual \\
\hline Revisión de conexiones eléctricas & Anual \\
\hline Apretar pernos y tornillos flojos & Anual \\
\hline $\begin{array}{l}\text { Buscar marcas de corrosión y desgaste en } \\
\text { rodamientos }\end{array}$ & Anual \\
\hline Engrase y ajuste de rodamiento de la torre & Anual \\
\hline
\end{tabular}




\begin{tabular}{|c|c|}
\hline Actividad & Periodicidad \\
\hline $\begin{array}{l}\text { Cambio de rodamientos del eje de trans- } \\
\text { misión }\end{array}$ & Depende del cojinete seleccionado \\
\hline Cambio de rodamiento de la torre & Depende del cojinete seleccionado \\
\hline $\begin{array}{l}\text { Cambio de recubrimiento en las } 3 \text { palas y } \\
\text { placa de veleta } \\
\text { Nota: remover la capa dañada por la } \\
\text { exposición al ambiente }\end{array}$ & $\begin{array}{l}\text { Cada } 10 \text { años (tomar consideraciones por } \\
\text { cada inspección rutinaria). }\end{array}$ \\
\hline \multicolumn{2}{|c|}{$\begin{array}{l}\text { Costo de mantenimiento estimado: entre } 1500.00-2000.00 \mathrm{RD} \$(25.71-34.28 \mathrm{USD}) \text {, por } \\
\text { cada cambio de rodamientos y recubrimiento de fibra de vidrio. } \\
\text { Nota: basado en la tabla de presupuesto. }\end{array}$} \\
\hline
\end{tabular}

Nota: tasa del dólar fue tomada como 58.35 RD\$ (julio 2020).

Este proyecto de diseño de palas mecánicas para aerogeneradores HAWT de $250 \mathrm{~W}$ se ha desarrollado dentro del INTEC, y puede considerarse como un proyecto piloto, como una primera parte de una serie de investigaciones orientadas a la parte mecánica de los aerogeneradores en lugares de bajos recursos. Este se presenta como un punto de partida, dando lugar para la mejora del diseño fortalecido en el análisis, prueba y experimentación con el prototipo que se fabrique.

\section{Conclusiones}

De acuerdo con la investigación realizada, el proyecto propuesto para el diseño mecánico de un aerogenerador de eje horizontal de 250 W constituye una opción viable y simple para las comunidades con escasez de energía eléctrica, por poseer una cantidad mínima de partes móviles. Teniendo siempre en cuenta que se debe realizar mediciones de viento para determinar la altura a la que se obtienen velocidad de viento de entre $10-11 \mathrm{~m} / \mathrm{s}$, que es donde se puede implementar este diseño que se apoya en los estudios de la NREL y las fórmulas del documento Introducción a teoría de las turbinas eólicas (Voneschen, 2009).

El prototipo reúne las características básicas y funcionales para su operación continua durante un tiempo de vida útil de 20 ańos, con un plan de mantenimiento preventivo de bajo coste, por tratarse de una 
máquina con pocas piezas móviles, tanto en las partes mecánicas como en el generador eléctrico diseñado en INTEC. El procedimiento de fabricación de moldes de madera de las palas por costillas es una de las propuestas con valor agregado al proyecto, el cual se convierte en una forma reutilizable y práctica para construir palas de aerogeneradores.

\section{Referencias}

AirfoilTools. (2020). Airfoil Tools. Disponible en http://airfoiltools.com/ Alsailani, M., Montazeri, H., \& Rezaeiha, A. (2021). Towards optimal aerodynamic design of wind catchers: Impact of geometrical characteristics. Renewable Energy, 168, 1344-1363. Disponible en https://doi.org/10.1016/j.renene.2020.12.053

Bernuzzi, C., Crespi, P., Montuori, R., Nastri, E., Simoncelli, M., Stochino, F., \& Zucca, M. (2021). Resonance of steel wind turbines: Problems and solutions. Structures, 32, 65-75. Disponible en https://doi.org/10.1016/j.istruc.2021.02.053

Budynas, R. G., \& Nisbett, J. K. (2012). Diseño en Ingeniería Mecánica de Shigley (9na ed.). Mexico: McGraw-Hill.

Cooperman, A., Eberle, A., \& Lantz, E. (2021). Wind turbine blade material in the United States: Quantities, costs, and end-of-life options. Resources, Conservation and Recycling, 168, 105439. Disponible en https://doi.org/10.1016/j.resconrec.2021.105439

Costoya, X., deCastro, M., Santos, F., Sousa, M. C., \& Gómez-Gesteira, M. (2019). Projections of wind energy resources in the Caribbean for the 21st century. Energy, 178, 356-367. Disponible en https://doi.org/10.1016/j.energy.2019.04.121

Denhof, D., Staar, B., Lütjen, M., \& Freitag, M. (2019). Automatic optical surface inspection of wind turbine rotor blades using convolutional neural networks. Procedia CIRP, 81, 1166-1170. Elsevier B.V. Disponible en https://doi.org/10.1016/j.procir. 2019.03.286

Dong, X., Li, J., Gao, D., \& Zheng, K. (2020). Wind speed modeling for cascade clusters of wind turbines part 1: The cascade clusters of wind turbines. Energy, 205, 118097. Disponible en https:// doi.org/10.1016/j.energy.2020.118097 
Elliott, D., Schwartz, M., George, R., Haymes, S., Heimiller, D., Scott, G., \& Kline, J. (2001). Wind Energy Resource Atlas of the Dominican Republic. Disponible en https://doi.org/NREL/TP-50027602

García, S., Herrera, J., Aristizábal, A. J., Castañeda, M., Zapata, S., \& Pelaez-Restrepo, J. (2019). Model of a wind turbine using discrete events. Energy Procedia, 157, 1029-1036. Elsevier Ltd. Disponible en https://doi.org/10.1016/j.egypro.2018.11.270

Gato, L. M. C., Maduro, A. R., Carrelhas, A. A. D., Henriques, J. C. C., $\&$ Ferreira, D. N. (2021). Performance improvement of the biradial self-rectifying impulse air-turbine for wave energy conversion by multi-row guide vanes: Design and experimental results. Energy, 216, 119110. Disponible en https://doi.org/10.1016/j. energy.2020.119110

Gugliani, G. K., Sarkar, A., Ley, C., \& Matsagar, V. (2021). Identification of optimum wind turbine parameters for varying wind climates using a novel month-based turbine performance index. Renewable Energy. Disponible en https://doi.org/10.1016/j. renene.2021.02.141

Herrmann, J., Rauert, T., Dalhoff, P., \& Sander, M. (2016). Fatigue and fracture mechanical behaviour of a wind turbine rotor shaft made of cast iron and forged steel. Procedia Structural Integrity, 2, 2951-2958. Elsevier B.V. Disponible en https://doi. org/10.1016/j.prostr.2016.06.369

Joustra, J., Flipsen, B., \& Balkenende, R. (2021). Structural reuse of high end composite products: A design case study on wind turbine blades. Resources, Conservation and Recycling, 167, 105393. Disponible en https://doi.org/10.1016/j.resconrec.2020.105393

Junginger, M., Hittinger, E., Williams, E., \& Wiser, R. (2019). Onshore wind energy. In Technological Learning in the Transition to a Low-Carbon Energy System: Conceptual Issues, Empirical Findings, and Use, in Energy Modeling (pp. 87-102). Elsevier. Disponible en https://doi.org/10.1016/B978-0-12-818762-3.00006-6

Lara, D. D., Merino, G. G., Pavez, B. J., \& Tapia, J. A. (2011). Efficiency assessment of a wind pumping system. Energy Conversion and Management, 52(2), 795-803. Disponible en https://doi. org/https://doi.org/10.1016/j.enconman.2010.08.004 
Li, Q., Maeda, T., Kamada, Y., \& Mori, N. (2017). Investigation of wake characteristics of a Horizontal Axis Wind Turbine in vertical axis direction with field experiments. Energy, 141, 262-272. Disponible en https://doi.org/10.1016/j.energy.2017.09.079

Lima Menjívar, F. J., Orellana Ramos, F. J., \& Valencia Gómez, E. B. (2017). Diseño y construcción de un prototipo de Aerogenerador de Baja Potencia (Universidad de El Salvador Universidad de El Salvador) San Salvador. Disponible en http://ri.ues.edu.sv/id/ eprint/13402/

López-Villalobos, C. A., Rodríguez-Hernández, O., Martínez-Alvarado, O., \& Hernández-Yepes, J. G. (2021). Effects of wind power spectrum analysis over resource assessment. Renewable Energy, 167,761-773. Disponible en https://doi.org/10.1016/j. renene.2020.11.147

Micha, P. T., Mohan, T., \& Sivamani, S. (2017). Design and Analysis of a Permanent Magnetic Bearing for Vertical Axis Small Wind Turbine. Energy Procedia, 117, 291-298. Elsevier Ltd. Disponible en https://doi.org/10.1016/j.egypro.2017.05.134

Moghadassian, B., \& Sharma, A. (2020). Designing wind turbine rotor blades to enhance energy capture in turbine arrays. Renewable Energy, 148, 651-664. Disponible en https://doi.org/10.1016/j. renene.2019.10.153

Mühle, F., Adaramola, M. S., \& Sætran, L. (2017). The effect of rotational direction on the wake of a wind turbine rotor-a comparison study of aligned co- and counter rotating turbine arrays. Energy Procedia, 137, 238-245. Elsevier Ltd. Disponible en https://doi. org/10.1016/j.egypro.2017.10.346

Ngbara Touafio, J. F., Sanda, O., Malenguinza, S., M’Boliguipa, J., \& Mouangue, R. M. (2020). Analysis of a wind turbine project in the city of Bouar (Central African Republic). Scientific African, 8, e00354. Disponible en https://doi.org/10.1016/j.sciaf.2020. e00354

Norton, R. L. (2009). Diseño de maquinaria. McGraw-Hill Interamericana de España S.L. Disponible en https://books.google.com. do/books?id=XOvPQwAACAAJ

Philpot, T. A. (2014). MDSolids. Disponible en https://web.mst. edu/ $\sim$ mdsolids/ 
Pomázi, Á., \& Toldy, A. (2021). Development of fire retardant epoxybased gelcoats for carbon fibre reinforced epoxy resin composites. Progress in Organic Coatings, 151, 106015. Disponible en https:// doi.org/10.1016/j.porgcoat.2020.106015

Rafiee, M., Nitzsche, F., Laliberte, J., Hind, S., Robitaille, F., \& Labrosse, M. R. (2019). Thermal properties of doubly reinforced fiberglass/epoxy composites with graphene nanoplatelets, graphene oxide and reduced-graphene oxide. Composites Part B: Engineering, 164, 1-9. Disponible en https://doi.org/10.1016/j. compositesb.2018.11.051

Rahimizadeh, A., Kalman, J., Fayazbakhsh, K., \& Lessard, L. (2019). Recycling of fiberglass wind turbine blades into reinforced filaments for use in Additive Manufacturing. Composites Part B: Engineering, 175, 107101. Disponible en https://doi.org/10.1016/j. compositesb.2019.107101

Rauert, T., Herrmann, J., Dalhoff, P., \& Sander, M. (2016). Fretting fatigue induced surface cracks under shrink fitted main bearings in wind turbine rotor shafts. Procedia Structural Integrity, 2, 36013609. Elsevier B.V. Disponible en https://doi.org/10.1016/j. prostr.2016.06.449

Rommel, D. P., Di Maio, D., \& Tinga, T. (2020). Calculating wind turbine component loads for improved life prediction. Renewable Energy, 146, 223-241. Disponible en https://doi.org/10.1016/j. renene.2019.06.131

Saint-Drenan, Y. M., Besseau, R., Jansen, M., Staffell, I., Troccoli, A., Dubus, L., ... Heier, S. (2020). A parametric model for wind turbine power curves incorporating environmental conditions. Renewable Energy, 157, 754-768. Disponible en https://doi. org/10.1016/j.renene.2020.04.123

Schaffarczyk, A. P. (2014). Introduction to Wind Turbine Aerodynamics. Springer Berlin Heidelberg. Retrieved from https://books.google. com.do/books?id=an0qBAAAQBAJ

Schmitz, S. (2020). Aerodynamics of Wind Turbines: A Physical Basis for Analysis and Design. Wiley. Disponible en https://books.google. com.do/books?id=RbzyDwAAQBAJ 
Sugathapala, T. M., Boteju, S., Withanage, P. B., \& Wijewardane, S. (2020). Aerodynamic modeling of simplified wind turbine rotors targeting small-scale applications in Sri Lanka. Energy for Sustainable Development, 59, 71-82. Disponible en https://doi. org/10.1016/j.esd.2020.09.004

Voneschen, M. F. (1989). Kleine Windräder: Berechnung und Konstruktion. Alemania: Verlag für Bauwesen. Disponible en https:// books.google.com.do/books?id=9auHAwAACAAJ

Voneschen, M. F. (2009). Introducción a la teoría de las turbinas eólicas 1. Retrieved June 25, 2021, from La veritat website: https:// docplayer.es/12407866-Introduccion-a-la-teoria-de-las-turbinas-eolicas-1.html

Xi, R., Wang, P., Du, X., Xu, K., Xu, C., \& Jia, J. (2020). A semianalytical model of aerodynamic damping for horizontal axis wind turbines and its applications. Ocean Engineering, 214, 107861. Disponible en https://doi.org/10.1016/j.oceaneng.2020.107861 